\title{
Links with trivial $Q$-polynomial
}

\author{
By Yasuyuki MrYazawa
}

(Received Jan. 19, 2017)

(Revised May 26, 2017)

\begin{abstract}
The $Q$-polynomial is an invariant of the isotopy type of an unoriented link defined by Brandt, Lickorish, Millett, and Ho around 1985. It is shown that there exist infinitely many prime knots and links with trivial $Q$-polynomial, and so the $Q$-polynomial does not detect trivial links.
\end{abstract}

\section{Introduction.}

"Does there exist a non-trivial knot indistinguishable from the trivial knot by the $Q$-polynomial?" It has been an open problem for 30 years since Brandt, Lickorish, Millett $[2]$ and Ho [6] introduced the $Q$-polynomial as an isotopy invariant for unoriented knots and links. In this paper, we give an affirmative answer. Furthermore, we reveal there exist infinitely many prime knots and links with trivial $Q$-polynomial; in this paper a polynomial invariant for a $\mu$-component link is trivial if the polynomial is identical with that of the trivial $\mu$-component link.

For a classical knot or link, there are several well-known polynomial invariants; the Alexander polynomial [1] is the most classical one whose geometric background is the infinite cyclic covering of a knot complement, and can be generalized to a $\mu$-variable Alexander polynomial for a $\mu$-component link. The Conway polynomial [3] is a version of the Alexander polynomial defined by the skein relation. The Jones polynomial [7] was discovered by Jones in 1984 using the braid theory, and soon after it was generalized to the HOMFLY polynomial [5], [19], [23], which is also specialized to the Alexander polynomial. On the other hand, considering an unoriented skein relation, around 1985 the $Q$-polynomial for unoriented knots and links was discovered by Brandt, Lickorish and Millett, which was independently found out by Ho around the same time. Then Kauffman generalized this to the Kauffman polynomial [12], which is also specialized to the Jones polynomial. See Figure 1, where an arrow indicates that one invariant produces another by means of a substitution of variables (cf. Figure 4 in [17]). In general, the Alexander, Jones and $Q$-polynomials have different topological features from one another. Two knots sharing one of three polynomials can be usually distinguished by the others unless they have a specific relation like mutants.

The existence of nontrivial knots or links with trivial Alexander polynomials, and the existence of nontrivial links (not a knot) with trivial Jones polynomials are known. The Alexander polynomial was defined in 1928. It is rather easy to construct an example of a nontrivial knot with trivial polynomial, e.g., the untwisted Whitehead double. In

2010 Mathematics Subject Classification. Primary 57M25; Secondary 57M27.

Key Words and Phrases. Q-polynomial, trivial polynomial. 


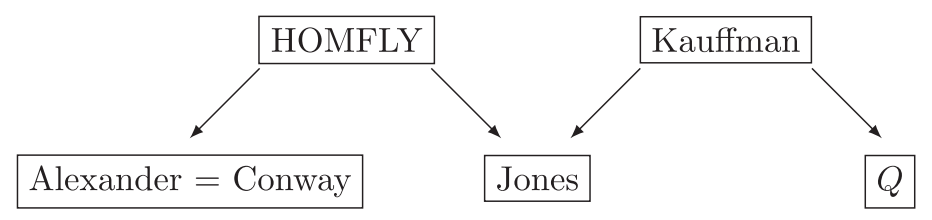

Figure 1. Relationships between five polynomials.

particular, the Kinoshita-Terasaka knot [14] and the Conway knot [3] are such examples with minimal crossing number. The Jones polynomial was discovered in 1985, and in 2001 Thistlethwaite [26] announced the existence of 2- and 3-component nontrivial links with trivial Jones polynomial by computer enumeration of links. Then, Eliahou, Kauffman and Thistlethwaite [4] constructed infinite families of links with trivial polynomials by a discovery of a pair of local moves which preserve the Jones polynomial. However, the problem of existence of a nontrivial knot with trivial Jones polynomial is still open. If the volume conjecture is true, then it turns out that any non-trivial knot has non-trivial Jones polynomial. Also, for the HOMFLY and Kauffman polynomials there are no known examples of links with trivial polynomials. However, Kanenobu [8] constructed infinitely many knots with the same Jones and HOMFLY polynomials.

The $Q$-polynomial $Q(L ; x) \in \mathbb{Z}\left[x^{ \pm 1}\right]$ for an unoriented link $L$ is an invariant of isotopy type of $L$, which is defined by the following recursive formulas:

(Q1) For the trivial knot $U_{1}, Q\left(U_{1} ; x\right)=1$,

(Q2) For a skein quadruple $\left(L_{+}, L_{-}, L_{0}, L_{\infty}\right)$ which denotes four links that are identical except near one point where they are as in Figure 2,

$$
Q\left(L_{+} ; x\right)+Q\left(L_{-} ; x\right)=x\left(Q\left(L_{0} ; x\right)+Q\left(L_{\infty} ; x\right)\right) .
$$

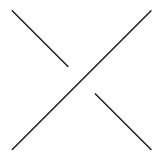

$L_{+}$

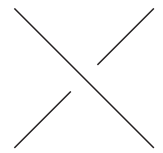

$L_{-}$
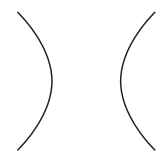

$L_{0}$

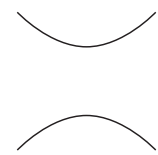

$L_{\infty}$

Figure 2. A skein quadruple.

Then, by simple calculation, we see that the $Q$-polynomial $Q\left(U_{\mu} ; x\right)$ for the trivial $\mu$-component link $U_{\mu}$ is $\left(2 x^{-1}-1\right)^{\mu-1}$.

The following is a conclusion on the problem mentioned at the beginning of the paper.

THEOREM 1.1. There exist non-trivial knots with trivial Q-polynomial.

Proof. We give explicit examples. The knots $16_{n 389841}$ and $16_{n 491778}$ illustrated in Figure 3 have trivial $Q$-polynomial. 


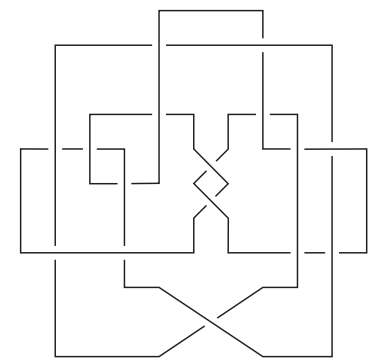

$16_{n 389841}$

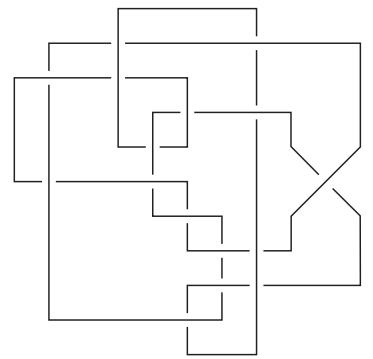

$16_{n 491778}$

Figure 3. Knots with trivial $Q$-polynomial.

Here the notation for the two knots above follows the Hoste-Thistlethwaite table of prime knots with up to 16 crossings.

The fact on the $Q$-polynomial in the proof of Theorem 1.1 is ensured by computations using two distinct tools, which are the program written by Morrison [21] in the Mathematica package "KnotTheory" and the software "KNOT" by Kodama [15], and hand calculation.

REMARK 1.2. Only the two knots above have trivial $Q$-polynomial among prime knots with up to 16 crossings. It is verified by examining non-alternating prime knots with up to 16 crossings using the Mathematica package "KnotTheory", because it is shown by Kidwell [13] and Miyauchi [20] that the degree of the $Q$-polynomial of an alternating knot is determined by its minimum crossing number.

Computing other classical polynomial invariants of the two knots above, we obtain a notable table.

Table 1. Triviality of polynomials.

\begin{tabular}{ccc}
\hline Knot & $16_{n 389841}$ & $16_{n 491778}$ \\
\hline$Q$ & trivial & trivial \\
Conway & non-trivial & trivial \\
Jones & non-trivial & non-trivial \\
\hline
\end{tabular}

Table 1 tells that the knot $16_{n 491778}$ has trivial Conway polynomial. Since the Jones polynomial of each knot is non-trivial, its HOMFLY and Kauffman polynomials are non-trivial.

Since, for any connected sum $L_{1} \sharp L_{2}$ of links $L_{1}$ and $L_{2}$, the $Q$-polynomial (resp. the Conway polynomial) of $L_{1} \sharp L_{2}$ is equal to the product of $Q$-polynomials (resp. Conway polynomials) of $L_{1}$ and $L_{2}$ [1], [2], by the unique factorization theorem [25], a composite link obtained from a given link and any number of copies of the knot $16_{n 491778}$ brings the following. 
COROLlary 1.3. For any link $L$, there exist infinitely many mutually distinct links with the same Conway and the same $Q$-polynomial as $L$.

Corollary 1.3 implies that the $Q$-polynomial dose not detect trivial links.

The purpose of this paper is to prove the following theorem.

THEOREM 1.4. For each positive integer $\mu$, there exist infinitely many mutually distinct prime $\mu$-component links with trivial Conway and $Q$-polynomials.

The proof is given by Theorems 2.5 and 2.6 which indicate that the specific knots and links introduced in the next section have the four features which are triviality of the two polynomials, mutually distinction between the links and primeness of the links. The theorems are completed by arguments and results on these features described in Section 3 and the following.

REMARK 1.5. As for prime links with the same $Q$-polynomial, Kanenobu [9] constructed arbitrarily many prime knots with the same $Q$-polynomial. Furthermore, he [10] constructed arbitrarily many 2-bridge knots and links with the same HOFMLY and Kauffman polynomials.

There is an interesting and important problem originating in Theorem 1.1.

Problem 1.6. Find a reason why the knots $16_{n 389841}$ and $16_{n 491778}$ have trivial Q-polynomial.

The author has no result on Problem 1.6 at this writing.

The rest of the paper subsequent to this section is devoted to the precise proof of Theorem 1.4 and organized as follows. In Section 2, a family of links is created for the proof of Theorem 1.4. Sections 3 and 4 explain that such links have trivial $Q$-polynomial and Conway polynomials, respectively. In Sections 5 and 6 , it is shown that the links are mutually distinct and prime, respectively.

\section{Knots and links for Theorem 1.4.}

In this section, we introduce the links with $\mu, \mu \geq 1$, components which are obtained from two specific tangles by a tangle sum and make two assertions on such links for proving Theorem 1.4.

A tangle $T$ is a pair $(B, t)$ of a 3-ball $B$ and a (possibly disconnected) proper 1submanifold $t$ with $\partial t \neq \emptyset$. $T$ is said to be a 2 -string tangle if $T$ ( $t$, to be precise) consists of two arcs and some circle components. Each of $\partial t$ is called an endpoint of $T$. The numerator (resp. denominator) of a 2-string tangle $T$ denoted by $N(T)$ (resp. $D(T)$ ) is a link obtained from $T$ by connecting the four endpoints of $T$ by two $\operatorname{arcs}$ outside $T$ as in the left (resp. right) figure of Figure 4.

We denote tangles as in Figure 5 by $E_{n}, n \in \mathbb{Z}, E_{\infty}$, and $E_{1 / m}, m \in \mathbb{Z}-\{0\}$, respectively. The tangle $E_{n}$ is a 2-string tangle with $|n|$ half-twists as drawn in Figure 5, right-handed for positive $n$ and left-handed for negative $n$; Figure 5 shows the righthanded twist. In particular, $E_{0}$ means horizontal parallel strings without crossings. We 

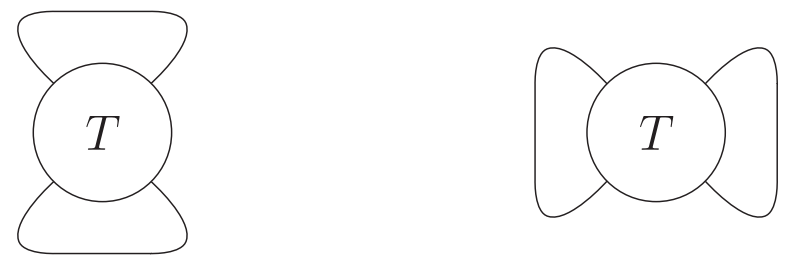

Figure 4. The numerator and the denominator.

denote by $E_{1 / m}$ the tangle obtained from $E_{m}$ by switching all crossings after rotating $E_{m}$ through angle $\pi / 2$ in the clockwise direction around an axis perpendicular to the projection plane. So, $E_{1 / m}$ has the twists depicted in Figure 5 if $m$ is positive, otherwise it has opposite twists.

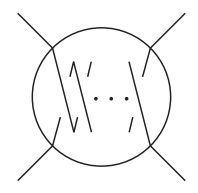

$E_{n}$

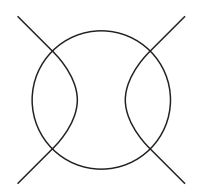

$E_{\infty}$

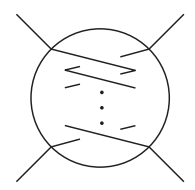

$E_{\frac{1}{m}}$

Figure 5. Trivial tangles.

Let $T$ and $S$ be 2-string tangles. We define addition of tangles $T$ and $S$ by connecting endpoints of $T$ and $S$ as in Figure 6 and denote it by $T+S$. We also define vertical addition of tangles $T$ and $S$ by connecting endpoints of $T$ and $S$ as in Figure 7 and denote it by $T * S$.

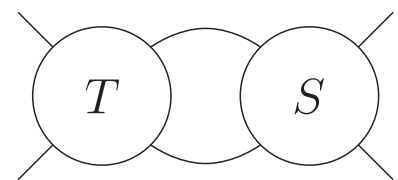

Figure 6. Addition of tangles.

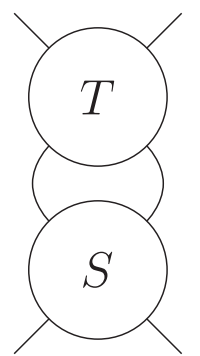

Figure 7. Vertical addition of tangles.

For positive integers $m$ and $n$, let $H(m, n)$ be the tangle $E_{1 /-m}+E_{1 / n}$ as in Figure 8. We denote by $R(m)$ the tangle $H(m, m+1) * H(m+1, m)$ which is the vertical addition of tangles $H(m, m+1)$ and $H(m+1, m)$. It is easy to see that there exists a mutation $\rho$ with respect to $R(m)$ such that $\rho(R(m))=\overline{R(m)}$.

Let $W$ be the tangle as in Figure 9 . Then, we have the following remark which is a key factor to obtain knots and links with trivial $Q$-polynomial. 


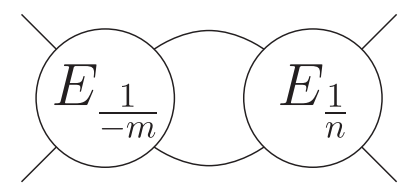

Figure 8. The tangle $H(m, n)$.

REMARK 2.1. The numerator and denominator of $W, N(W)$ and $D(W)$, are the trivial 2-component link and the knot $16_{n 491778}$, respectively.

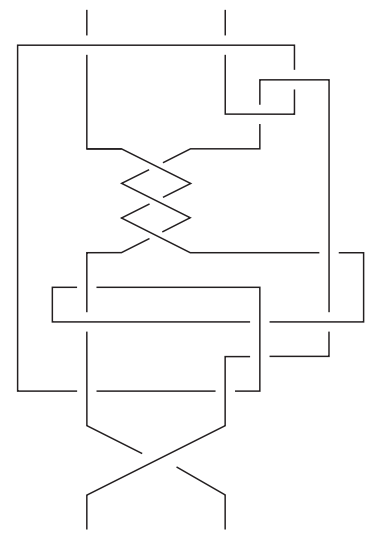

Figure 9. The tangle $W$.

For a positive integer $m$, we denote the knot $N(R(m)+W)$ by $K_{m}$.

ExAmple 2.2. Figure 10 displays a diagram of the knot $K_{2}=N(R(2)+W)$.

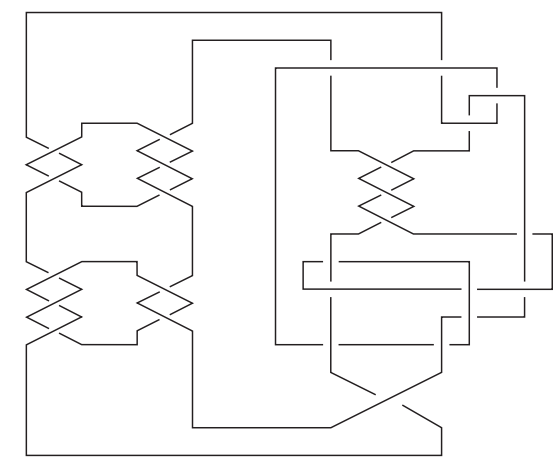

Figure 10. $\quad K_{2}=N(R(2)+W)$.

For an integer $\mu \geq 2$, we denote by $S(\mu ; m)$ the tangle $H(2 m, 2 m) *\left(*_{\mu-2} H(2,2)\right)$, which is the vertical addition of $H(2 m, 2 m)$ and $(\mu-2)$ copies of $H(2,2)$. We also define 
the link $N(S(\mu ; m)+W)$ by $L_{(\mu ; m)}$.

EXAMPLE 2.3. Figure 11 demonstrates a diagram of the link $L_{(3 ; 2)}=N(S(3 ; 2)+$ $W)$.

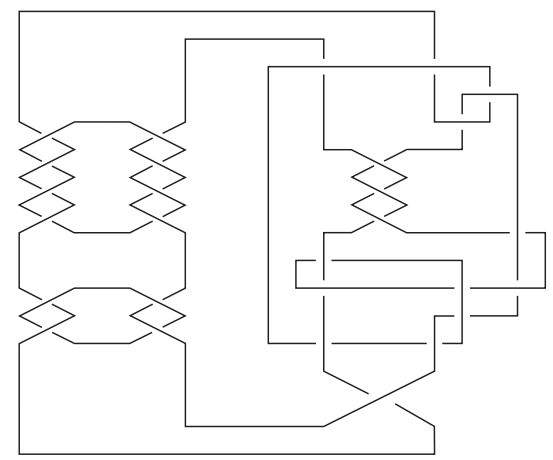

Figure 11. $\quad L_{(3 ; 2)}=N(S(3 ; 2)+W)$.

Next, we orient the knot $K_{m}$ and the link $L_{(\mu ; m)}$.

An oriented 2-string tangle $T$ is called properly oriented if each arc of $T$ is oriented as in Figure 12. If $T$ is properly oriented, then we suppose that the links $N(T)$ and $D(T)$ have the orientations induced from $T$.

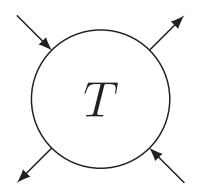

Figure 12. A properly oriented 2-string tangle.

REMARK 2.4. If two tangles $T$ and $S$ are properly oriented, then each of tangles $T+S$ and $T * S$ is also properly oriented.

The tangle $R(m)$ is a 2-string tangle without circle components, and one of the strings of $R(m)$ connects the two endpoints on the left side of the tangle and the other connects the two endpoints on the right side. Thus, we can orient $R(m)$ such that it becomes properly oriented. We can also orient the tangle $W$ as it is properly oriented. From the two properly oriented tangles $R(m)$ and $W$, we can obtain the oriented knot $K_{m}=N(R(m)+W)$.

The tangle $H(m, n)=E_{1 /-m}+E_{1 / n}, m, n \in \mathbb{N}$, can be oriented as follows:

(1) $H(m, n)$ is properly oriented,

(2) Each of $E_{1 /-m}$ and $E_{1 / n}$ is oriented so that two strings of the tangle have parallel orientations. 
Then, the tangle $S(\mu ; m), m \in \mathbb{N}$, becomes a property oriented one. From the two properly oriented tangles $S(\mu ; m)$ and $W$, we can obtain the oriented link $L_{(\mu ; m)}=$ $N(S(\mu ; m)+W)$.

So, if necessary, we suppose that $K_{m}$ and $L_{(\mu ; m)}$ are oriented as mentioned above.

TheOREM 2.5. The knots $K_{m}, m \in \mathbb{N}$, satisfy the following:

(1) $Q\left(K_{m} ; x\right)=Q\left(U_{1} ; x\right)=1$,

(2) $\nabla\left(K_{m} ; z\right)=\nabla\left(U_{1} ; z\right)=1$,

(3) $K_{m}$ is prime,

(4) $K_{m}$ and $K_{n}$ are distinct if $m \neq n$,

where $\nabla(L ; z)$ denotes the Conway polynomial of a link $L$ mentioned in Section 1 and defined precisely in Section 4.

Proof. The proof is accomplished by Corollaries 3.8, 4.2, 5.16 and 6.8.

TheOREM 2.6. The links $L_{(\mu ; m)}, \mu \geq 2, m \geq 1$, satisfy the following:

(1) $Q\left(L_{(\mu ; m)} ; x\right)=Q\left(U_{\mu} ; x\right)=\left(2 x^{-1}-1\right)^{\mu-1}$,

(2) $\nabla\left(L_{(\mu ; m)} ; z\right)=\nabla\left(U_{\mu} ; z\right)=0$,

(3) $L_{(\mu ; m)}$ is prime,

(4) $L_{(\mu ; m)}$ and $L_{(\mu ; n)}$ are distinct if $m \neq n$.

Proof. The proof is completed by Corollaries 3.9, 4.2, 5.19, 5.20 and 6.8 .

It is easy to see that Theorems 2.5 and 2.6 imply Theorem 1.4. The corollaries which yields Theorems 2.5 and 2.6 are given in the following sections.

\section{Trivial $Q$-polynomial.}

In this section, we show that $K_{m}$ and $L_{(\mu ; m)}$ defined in the previous section have trivial $Q$-polynomial. First, we examine the $Q$-polynomial of a link obtained from two tangles by a tangle sum. Next, we apply a result to creation of links with trivial $Q$-polynomial. For the sake of argument, we prepare Proposition 3.2 which reveals characteristics of the $Q$-polynomial.

Let $3_{1}$ be the trefoil knot in the table of Rolfsen $[\mathbf{2 4}]$. We define a polynomial $r_{\mu}(x) \in \mathbb{Z}\left[x^{ \pm 1}\right]$ for $\mu \in \mathbb{N}$ as follows:

(1) $r_{1}(x)=Q\left(3_{1} ; x\right)-Q\left(U_{1} ; x\right)=2(x+2)(x-1)$,

(2) $r_{\mu}(x)=x^{1-\mu} r_{1}(x)$ if $\mu \geq 2$.

LEMma 3.1. For an integer $\mu$ with $\mu>1$,

$$
2 Q\left(U_{\mu} ; x\right)-2 x Q\left(U_{\mu-1} ; x\right)+r_{\mu}(x)(2-x)^{\mu-2}=0 .
$$


Proof. Since $Q\left(U_{\mu} ; x\right)=Q\left(U_{2} ; x\right)^{\mu-1}$ and $r_{1}(x)=2\left(x^{2}+x-2\right)$, we obtain

$$
\begin{aligned}
2 Q\left(U_{\mu} ; x\right)-2 x Q\left(U_{\mu-1} ; x\right) & =2\left(Q\left(U_{2} ; x\right)^{\mu-1}-x Q\left(U_{2} ; x\right)^{\mu-2}\right) \\
& =2\left(Q\left(U_{2} ; x\right)-x\right) Q\left(U_{2} ; x\right)^{\mu-2} \\
& =2\left(2 x^{-1}-1-x\right) Q\left(U_{2} ; x\right)^{\mu-2} \\
& =-x^{-1} r_{1}(x)\left\{x^{-1}(2-x)\right\}^{\mu-2} \\
& =-r_{\mu}(x)(2-x)^{\mu-2} .
\end{aligned}
$$

This completes the proof.

For a link $L$, we denote the number of components of $L$ by $\mu(L)$.

Proposition 3.2. Let $L$ be a link. Then,

$$
Q(L ; x) \equiv Q\left(U_{\mu(L)} ; x\right) \quad\left(\bmod r_{\mu(L)}(x)\right) .
$$

Furthermore, $\widehat{Q}(L ; x) \in \mathbb{Z}[x]$, where

$$
\widehat{Q}(L ; x)=\frac{Q(L ; x)-Q\left(U_{\mu(L)} ; x\right)}{r_{\mu(L)}(x)} .
$$

Proof. The proof is by induction on $(c(L), c h(L))$, where $c(L)$ is the number of the crossings of a diagram of $L$ and $\operatorname{ch}(L)$ is the number of crossing changes needed to obtain the trivial link with the same component as $L$. The pairs $(c(L), \operatorname{ch}(L))$ are ordered lexicographically. If $(c(L), \operatorname{ch}(L))=(m, 0), m \geq 0$, then the claim is true. Suppose that $(c(L), \operatorname{ch}(L))=(m, n), 1 \leq n \leq m$. Then, there exists an $n$-tuple $\left(c_{1}, c_{2}, \ldots, c_{n}\right)$ of crossings $c_{1}, c_{2}, \ldots, c_{n}$ of $L$ such that $U_{\mu(L)}$ is obtained from $L$ by changing all crossings of the $n$-tuple. We choose any crossing from the $n$-tuple and denote it by $c$. We denote by $S_{c} L$ the link obtained from $L$ by switching $c$ and also denote by $Z_{c} L$ (resp. $I_{c} L$ ) the link obtained from $L$ by applying 0 -splice (resp. $\infty$-splice) at $c$, where 0 -splice (resp. $\infty$-splice) means an operation obtaining $L_{0}$ (resp. $\left.L_{\infty}\right)$ from $L_{+}$as in Figure 2. Then, we have

$$
Q(L ; x)+Q\left(S_{c} L ; x\right)=x\left(Q\left(Z_{c} L ; x\right)+Q\left(I_{c} L ; x\right)\right) .
$$

Since a skein quadruple $\left(L_{+}, L_{-}, L_{0}, L_{\infty}\right)$ can be regarded as a skein quadruple $\left(L_{-}, L_{+}, L_{\infty}, L_{0}\right)$, we may assume that $\mu(L) \neq \mu\left(Z_{c} L\right)$. Hence, we have two cases according to the numbers $\mu(L)$ and $\mu\left(Z_{c} L\right)$.

First, we consider the case $\mu(L)<\mu\left(Z_{c} L\right)$. Then, $\mu(L)=\mu\left(S_{c} L\right)=\mu\left(I_{c} L\right)=$ $\mu\left(Z_{c} L\right)-1$. Since the three pairs $\left(c\left(S_{c} L\right), \operatorname{ch}\left(S_{c} L\right)\right),\left(c\left(Z_{c} L\right), \operatorname{ch}\left(Z_{c} L\right)\right)$ and $\left(c\left(I_{c} L\right)\right.$, $\left.\operatorname{ch}\left(I_{c} L\right)\right)$ are less than the pair $(c(L), \operatorname{ch}(L))$, by inductive hypothesis, we have

$$
\begin{aligned}
Q\left(S_{c} L ; x\right) & =Q\left(U_{\mu} ; x\right)+r_{\mu}(x) \widehat{Q}\left(S_{c} L ; x\right), \\
Q\left(I_{c} L ; x\right) & =Q\left(U_{\mu} ; x\right)+r_{\mu}(x) \widehat{Q}\left(I_{c} L ; x\right),
\end{aligned}
$$


and

$$
Q\left(Z_{c} L ; x\right)=Q\left(U_{\mu+1} ; x\right)+r_{\mu+1}(x) \widehat{Q}\left(Z_{c} L ; x\right),
$$

where $\mu=\mu(L)$ and $\widehat{Q}\left(S_{c} L ; x\right), \widehat{Q}\left(I_{c} L ; x\right), \widehat{Q}\left(Z_{c} L ; x\right) \in \mathbb{Z}[x]$. Hence,

$$
\begin{aligned}
Q(L ; x)= & -Q\left(S_{c} L ; x\right)+x\left(Q\left(Z_{c} L ; x\right)+Q\left(I_{c} L ; x\right)\right) \\
= & -Q\left(U_{\mu} ; x\right)-r_{\mu}(x) \widehat{Q}\left(S_{c} L ; x\right) \\
& +x\left(Q\left(U_{\mu+1} ; x\right)+r_{\mu+1}(x) \widehat{Q}\left(Z_{c} L ; x\right)+Q\left(U_{\mu} ; x\right)+r_{\mu}(x) \widehat{Q}\left(I_{c} L ; x\right)\right) \\
= & \left\{-Q\left(U_{\mu} ; x\right)+x\left(Q\left(U_{\mu+1} ; x\right)+Q\left(U_{\mu} ; x\right)\right)\right\} \\
& -r_{\mu}(x) \widehat{Q}\left(S_{c} L ; x\right)+x r_{\mu+1}(x) \widehat{Q}\left(Z_{c} L ; x\right)+x r_{\mu}(x) \widehat{Q}\left(I_{c} L ; x\right) \\
= & Q\left(U_{\mu} ; x\right)+r_{\mu}(x)\left(-\widehat{Q}\left(S_{c} L ; x\right)+\widehat{Q}\left(Z_{c} L ; x\right)+x \widehat{Q}\left(I_{c} L ; x\right)\right) .
\end{aligned}
$$

Since $-\widehat{Q}\left(S_{c} L ; x\right)+\widehat{Q}\left(Z_{c} L ; x\right)+x \widehat{Q}\left(I_{c} L ; x\right) \in \mathbb{Z}[x]$, the claim is true.

Next, we consider the case $\mu(L)>\mu\left(Z_{c} L\right)$. Note that $\mu(L)>1$. It is clear that $\mu(L)=\mu\left(S_{c} L\right)=\mu\left(Z_{c} L\right)+1=\mu\left(I_{c} L\right)+1$. Since inductive hypothesis gives

$$
\begin{aligned}
& Q\left(S_{c} L ; x\right)=Q\left(U_{\mu} ; x\right)+r_{\mu}(x) \widehat{Q}\left(S_{c} L ; x\right), \\
& Q\left(Z_{c} L ; x\right)=Q\left(U_{\mu-1} ; x\right)+r_{\mu-1}(x) \widehat{Q}\left(Z_{c} L ; x\right),
\end{aligned}
$$

and

$$
Q\left(I_{c} L ; x\right)=Q\left(U_{\mu-1} ; x\right)+r_{\mu-1}(x) \widehat{Q}\left(I_{c} L ; x\right),
$$

where $\mu=\mu(L)$ and $\widehat{Q}\left(S_{c} L ; x\right), \widehat{Q}\left(I_{c} L ; x\right), \widehat{Q}\left(Z_{c} L ; x\right) \in \mathbb{Z}[x]$, we obtain

$$
\begin{aligned}
Q(L ; x)=- & Q\left(S_{c} L ; x\right)+x\left(Q\left(Z_{c} L ; x\right)+Q\left(I_{c} L ; x\right)\right) \\
=- & Q\left(U_{\mu} ; x\right)-r_{\mu}(x) \widehat{Q}\left(S_{c} L ; x\right) \\
& +x\left(2 Q\left(U_{\mu-1} ; x\right)+r_{\mu-1}(x) \widehat{Q}\left(Z_{c} L ; x\right)+r_{\mu-1}(x) \widehat{Q}\left(I_{c} L ; x\right)\right) \\
= & \left.-Q\left(U_{\mu} ; x\right)+2 x Q\left(U_{\mu-1} ; x\right)\right\} \\
& +r_{\mu}(x)\left\{-\widehat{Q}\left(S_{c} L ; x\right)+x^{2}\left(\widehat{Q}\left(Z_{c} L ; x\right)+\widehat{Q}\left(I_{c} L ; x\right)\right)\right\} .
\end{aligned}
$$

Since $-Q\left(U_{\mu} ; x\right)+2 x Q\left(U_{\mu-1} ; x\right)=Q\left(U_{\mu} ; x\right)+r_{\mu}(x)(2-x)^{\mu-2}$ by Lemma 3.1 , the last expression is equal to

$$
Q\left(U_{\mu} ; x\right)+r_{\mu}(x)\left\{-\widehat{Q}\left(S_{c} L ; x\right)+x^{2}\left(\widehat{Q}\left(Z_{c} L ; x\right)+\widehat{Q}\left(I_{c} L ; x\right)\right)+(2-x)^{\mu-2}\right\} .
$$

Since $-\widehat{Q}\left(S_{c} L ; x\right)+x^{2}\left(\widehat{Q}\left(Z_{c} L ; x\right)+\widehat{Q}\left(I_{c} L ; x\right)\right)+(2-x)^{\mu-2} \in \mathbb{Z}[x]$ for $\mu>1$, the proof is complete.

We call the polynomial $\widehat{Q}(L ; x)$ the core $Q$-polynomial of $L$. Since the $Q$-polynomial is an invariant for a link, the core $Q$-polynomial $\widehat{Q}(L ; x)$ is also an invariant for a link. If 
$L$ has trivial $Q$-polynomial, then the core $Q$-polynomial of $L$ vanishes by the definition.

A close look at the proof of Proposition 3.2 makes the following clear.

Corollary 3.3. The core $Q$-polynomial $\widehat{Q}(L ; x)$ for an unoriented link $L$ is given by the following recursive formulas:

$(\widehat{Q} 1)$ For the trivial knot $U_{1}, \widehat{Q}\left(U_{1} ; x\right)=0$,

$(\widehat{Q} 2)$ For a skein quadruple $\left(L_{+}, L_{-}, L_{0}, L_{\infty}\right)$ with $\mu\left(L_{+}\right) \neq \mu\left(L_{0}\right)$,

$$
\begin{aligned}
\widehat{Q}\left(L_{+} ; x\right) & +\widehat{Q}\left(L_{-} ; x\right) \\
& = \begin{cases}\widehat{Q}\left(L_{0} ; x\right)+x \widehat{Q}\left(L_{\infty} ; x\right) & \text { if } \mu\left(L_{+}\right)<\mu\left(L_{0}\right), \\
x^{2}\left(\widehat{Q}\left(L_{0} ; x\right)+\widehat{Q}\left(L_{\infty} ; x\right)\right)+(2-x)^{\mu\left(L_{+}\right)-2} & \text { if } \mu\left(L_{+}\right)>\mu\left(L_{0}\right) .\end{cases}
\end{aligned}
$$

From the proof of Proposition 3.2, we find that two formulas ( $\widehat{Q} 1)$ and $(\widehat{Q} 2)$ hold. To prove the corollary, it is enough to show that the core $Q$-polynomial can be computed by the two formulas only. It can be easily accomplished by induction on the pair $(c(L), \operatorname{ch}(L))$. We omit the details here.

Let $L(T), L\left(E_{0}\right), L\left(E_{\infty}\right)$ and $L\left(E_{-1}\right)$ be four links identical outside a ball and inside are 2-string tangles $T, E_{0}, E_{\infty}$ and $E_{-1}$, respectively. Then, linear skein theory (cf. [18]) gives a unique triple $\left(e_{0}(T ; x), e_{\infty}(T ; x), e_{-1}(T ; x)\right)$ of Laurent polynomials in $x$ for the tangle $T$ so that

$$
\begin{aligned}
Q(L(T) ; x)=e_{0} & (T ; x) Q\left(L\left(E_{0}\right) ; x\right) \\
& +e_{\infty}(T ; x) Q\left(L\left(E_{\infty}\right) ; x\right)+e_{-1}(T ; x) Q\left(L\left(E_{-1}\right) ; x\right) .
\end{aligned}
$$

The polynomials $e_{0}(T ; x), e_{\infty}(T ; x)$ and $e_{-1}(T ; x)$ are essentially determined by the tangle $T$ only. We call the triple $\left(e_{0}(T ; x), e_{\infty}(T ; x), e_{-1}(T ; x)\right)$ the coordinates of $T$ on the $Q$ polynomial with respect to basic tangles $E_{0}, E_{\infty}$ and $E_{-1}$.

Let $M_{1}$ and $M_{2}$ be $3 \times 3$ matrices whose entries are in $\mathbb{Z}\left[x^{ \pm 1}\right]$ defined by

$$
\left(\begin{array}{ccc}
2 x^{-1}-1 & 1 & 1 \\
1 & 2 x^{-1}-1 & 1 \\
1 & 1 & 2 x^{-1}-1
\end{array}\right) \text { and }\left(\begin{array}{ccc}
-2 x & x^{2} & x^{2} \\
x^{2} & -2 x & x^{2} \\
x^{2} & x^{2} & -2 x
\end{array}\right) \text {, respectively. }
$$

Lemma 3.4. Let $T$ be a 2-string tangle and a triple $\left(e_{0}(T ; x), e_{\infty}(T ; x), e_{-1}(T ; x)\right)$ of polynomials the coordinates of $T$ on the $Q$-polynomial with respect to basic tangles $E_{0}, E_{\infty}$ and $E_{-1}$. Then,

$$
\left(\begin{array}{c}
e_{0}(T ; x) \\
e_{\infty}(T ; x) \\
e_{-1}(T ; x)
\end{array}\right)=\frac{1}{r_{1}(x)} M_{2}\left(\begin{array}{c}
Q(N(T) ; x) \\
Q(D(T) ; x) \\
Q\left(N\left(T+E_{1}\right) ; x\right)
\end{array}\right)
$$

Proof. Considering $N(T), D(T)$ and $N\left(T+E_{-1}\right)$ as $L(T)$ in Equation (3.1), we have 


$$
\begin{aligned}
\left(\begin{array}{c}
Q(N(T) ; x) \\
Q(D(T) ; x) \\
Q\left(N\left(T+E_{1}\right) ; x\right)
\end{array}\right) & =\left(\begin{array}{c}
Q\left(U_{2} ; x\right) Q\left(U_{1} ; x\right) Q\left(U_{1} ; x\right) \\
Q\left(U_{1} ; x\right) Q\left(U_{2} ; x\right) Q\left(U_{1} ; x\right) \\
Q\left(U_{1} ; x\right) Q\left(U_{1} ; x\right) Q\left(U_{2} ; x\right)
\end{array}\right)\left(\begin{array}{c}
e_{0}(T ; x) \\
e_{\infty}(T ; x) \\
e_{-1}(T ; x)
\end{array}\right) \\
& =M_{1}\left(\begin{array}{c}
e_{0}(T ; x) \\
e_{\infty}(T ; x) \\
e_{-1}(T ; x)
\end{array}\right) .
\end{aligned}
$$

Since $M_{2} M_{1}=r_{1}(x) I_{3}$, where $I_{n}, n \in \mathbb{N}$, denotes the identity matrix of size $n$, we have

$$
r_{1}(x)\left(\begin{array}{c}
e_{0}(T ; x) \\
e_{\infty}(T ; x) \\
e_{-1}(T ; x)
\end{array}\right)=M_{2}\left(\begin{array}{c}
Q(N(T) ; x) \\
Q(D(T) ; x) \\
Q\left(N\left(T+E_{1}\right) ; x\right)
\end{array}\right)
$$

completing the proof.

For a matrix $X$, we denote its transpose by ${ }^{t} X$.

Proposition 3.5. Let $S$ and $T$ be 2-string tangles. Suppose that $T$ has no circle components. Then,

$$
Q(N(S+T) ; x)=\left(\begin{array}{c}
Q(N(S) ; x) \\
Q(D(S) ; x) \\
Q\left(N\left(S+E_{-1}\right) ; x\right)
\end{array}\right)\left(A\left(\begin{array}{c}
\widehat{Q}(N(T) ; x) \\
\widehat{Q}(D(T) ; x) \\
\widehat{Q}\left(N\left(T+E_{1}\right) ; x\right)
\end{array}\right)+B\right)
$$

where the pair $(A, B)$ of matrices is as follows:

(1) If $\mu(N(T))=2$, then $(A, B)=\left(\left(\begin{array}{ccc}-2 & x^{2} & x^{2} \\ x & -2 x & x^{2} \\ x & x^{2} & -2 x\end{array}\right),\left(\begin{array}{l}1 \\ 0 \\ 0\end{array}\right)\right)$,

(2) If $\mu(D(T))=2$, then $(A, B)=\left(\left(\begin{array}{ccc}-2 x & x & x^{2} \\ x^{2} & -2 & x^{2} \\ x^{2} & x & -2 x\end{array}\right),\left(\begin{array}{l}0 \\ 1 \\ 0\end{array}\right)\right)$,

(3) If $\mu\left(N\left(T+E_{1}\right)\right)=2$, then $(A, B)=\left(\left(\begin{array}{ccc}-2 x & x^{2} & x \\ x^{2} & -2 x & x \\ x^{2} & x^{2} & -2\end{array}\right),\left(\begin{array}{l}0 \\ 0 \\ 1\end{array}\right)\right)$.

Proof. We prove the first case only because the others can be proved similarly. Put $C(T)={ }^{t}\left(e_{0}(T ; x) e_{\infty}(T ; x) e_{-1}(T ; x)\right)$. Considering decomposition of $T$ by Equation (3.1), we have

$$
\begin{aligned}
Q(N(S+T) ; x)= & e_{0}(T ; x) Q\left(N\left(S+E_{0}\right) ; x\right)+e_{\infty}(T ; x) Q\left(N\left(S+E_{\infty}\right) ; x\right) \\
& +e_{-1}(T ; x) Q\left(N\left(S+E_{-1}\right) ; x\right) \\
= & e_{0}(T ; x) Q(N(S) ; x)+e_{\infty}(T ; x) Q(D(S) ; x) \\
& +e_{-1}(T ; x) Q\left(N\left(S+E_{-1}\right) ; x\right)
\end{aligned}
$$




$$
=\left(Q(N(S) ; x) Q(D(S) ; x) Q\left(N\left(S+E_{-1}\right) ; x\right)\right) C(T) .
$$

Thus, we only have to calculate $C(T)$ by using Lemma 3.4 .

Since $\mu(N(T))=2$, we find that $\mu(D(T))=\mu\left(N\left(T+E_{1}\right)\right)=1$. Using Proposition 3.2 , we obtain

$$
\begin{aligned}
& \left(\begin{array}{c}
Q(N(T) ; x) \\
Q(D(T) ; x) \\
Q\left(N\left(T+E_{1}\right) ; x\right)
\end{array}\right)=\left(\begin{array}{c}
x^{-1} \widehat{Q}(N(T) ; x) r_{1}(x)+\left(2 x^{-1}-1\right) \\
\widehat{Q}(D(T) ; x) r_{1}(x)+1 \\
\widehat{Q}\left(N\left(T+E_{1}\right) ; x\right) r_{1}(x)+1
\end{array}\right) \\
& =r_{1}(x)\left(\begin{array}{ccc}
x^{-1} & 0 & 0 \\
0 & 1 & 0 \\
0 & 0 & 1
\end{array}\right)\left(\begin{array}{c}
\widehat{Q}(N(T) ; x) \\
\widehat{Q}(D(T) ; x) \\
\widehat{Q}\left(N\left(T+E_{1}\right) ; x\right)
\end{array}\right)+\left(\begin{array}{c}
2 x^{-1}-1 \\
1 \\
1
\end{array}\right) .
\end{aligned}
$$

Since

$$
M_{2}\left(\begin{array}{ccc}
x^{-1} & 0 & 0 \\
0 & 1 & 0 \\
0 & 0 & 1
\end{array}\right)=\left(\begin{array}{ccc}
-2 & x^{2} & x^{2} \\
x & -2 x & x^{2} \\
x & x^{2} & -2 x
\end{array}\right) \quad \text { and } \quad M_{2}\left(\begin{array}{c}
2 x^{-1}-1 \\
1 \\
1
\end{array}\right)=r_{1}(x)\left(\begin{array}{l}
1 \\
0 \\
0
\end{array}\right)
$$

Lemma 3.4 gives the claim.

For a tangle $T$, we denote by $\bar{T}$ the mirror image of $T$ with respect to projection plane, that is the tangle obtained from $T$ by switching all crossings in $T$.

A mutation $\rho$ with respect to a tangle $T$ is one of the rotations of $\pi / 180^{\circ}$ about one of its three principal axes and we denote by $\rho(T)$ the tangle obtained from $T$ by a mutation $\rho$ with respect to $T$.

For a link $L, L$ ! denotes the mirror image of $L$ in a usual sense.

TheOrem 3.6. Let $R$ and $T$ be 2-string tangles. Suppose that $R$ and $T$ satisfy the following:

(1) There exists a mutation $\rho$ with respect to $R$ such that $\rho(R)=\bar{R}$,

(2) $N(T)$ is a 2-component link with trivial Q-polynomial,

(3) $D(T)$ is a knot with trivial Q-polynomial.

Then,

$$
Q(N(R+T) ; x)=Q(N(R) ; x) .
$$

Proof. Since $D(T)$ is a knot, $T$ has no circle components. Since $N(T)$ has two components, Proposition 3.5 gives

$$
Q(N(R+T) ; x)=\left(\begin{array}{c}
Q(N(R) ; x) \\
Q(D(R) ; x) \\
Q\left(N\left(R+E_{-1}\right) ; x\right)
\end{array}\right)\left(M\left(\begin{array}{c}
\widehat{Q}(N(T) ; x) \\
\widehat{Q}(D(T) ; x) \\
\widehat{Q}\left(N\left(T+E_{1}\right) ; x\right)
\end{array}\right)+\left(\begin{array}{l}
1 \\
0 \\
0
\end{array}\right)\right)
$$

where 


$$
M=\left(\begin{array}{ccc}
-2 & x^{2} & x^{2} \\
x & -2 x & x^{2} \\
x & x^{2} & -2 x
\end{array}\right)
$$

Since $Q(N(T) ; x)=Q\left(U_{2} ; x\right)$ and $Q(D(T) ; x)=Q\left(U_{1} ; x\right)$ by the assumption, we have $\widehat{Q}(N(T) ; x)=\widehat{Q}(D(T) ; x)=0$ and thus,

$$
\begin{aligned}
Q(N(R+T) ; x)=Q & (N(R) ; x)+x \widehat{Q}\left(N\left(T+E_{1}\right) ; x\right) \\
& \times\left\{x(Q(N(R) ; x)+Q(D(R) ; x))-2 Q\left(N\left(R+E_{-1}\right) ; x\right)\right\} .
\end{aligned}
$$

We show that $x(Q(N(R) ; x)+Q(D(R) ; x))-2 Q\left(N\left(R+E_{-1}\right) ; x\right)=0$. Since the tangle $\rho\left(R+E_{1}\right)$ is either the tangle $E_{1}+\bar{R}$ or the tangle $\bar{R}+E_{1}$, and $N\left(E_{1}+\bar{R}\right)$ and $N\left(\bar{R}+E_{1}\right)$ are ambient isotopic, three links $N\left(\rho\left(R+E_{1}\right)\right), N\left(\bar{R}+E_{1}\right)$ and $N\left(R+E_{-1}\right)$ ! are ambient isotopic and

$$
\begin{aligned}
Q\left(N\left(R+E_{1}\right) ; x\right) & =Q\left(N\left(\rho\left(R+E_{1}\right)\right) ; x\right)=Q\left(N\left(R+E_{-1}\right) ! ; x\right) \\
& =Q\left(N\left(R+E_{-1}\right) ; x\right),
\end{aligned}
$$

because the $Q$-polynomial dose not distinguish a link from its mirror image. Hence, by the skein relation $(\mathrm{Q} 2)$,

$$
\begin{aligned}
2 Q\left(N\left(R+E_{-1}\right) ; x\right)-x(Q(N(R) ; x)+Q(D(R) ; x)) \\
=Q\left(N\left(R+E_{1}\right) ; x\right)+Q\left(N\left(R+E_{-1}\right) ; x\right) \\
\quad-x\left(Q\left(N\left(R+E_{0}\right) ; x\right)+Q\left(N\left(R+E_{\infty}\right) ; x\right)\right) \\
=0 .
\end{aligned}
$$

This completes the proof.

REMARK 3.7. From an observation on the proof of Theorem 3.6, we may replace the condition (1) on the tangle $R$ with the condition $Q\left(N\left(R+E_{1}\right) ; x\right)=Q\left(N\left(R+E_{-1}\right) ; x\right)$ on the $Q$-polynomial.

Now we create links with trivial $Q$-polynomial. We consider the tangles $R(m), S(\mu, m)$ and $W$ defined in Section 2. We already mentioned that there exists a mutation $\rho$ with respect to $R(m)$ such that $\rho(R(m))=\overline{R(m)}$. Remark 2.1 gives $Q(N(W) ; x)=Q\left(U_{2} ; x\right)$ and $Q(D(W) ; x)=Q\left(U_{1} ; x\right)$. Since $N(R(m))$ is the trivial knot, we have $Q(N(R(m)) ; x)=1$. Then, applying Theorem 3.6, we obtain the following.

Corollary 3.8. $Q\left(K_{m} ; x\right)=1$.

It is obvious that there exists a mutation $\rho$ with respect to $S(\mu ; m)$ such that $\rho(S(\mu ; m))=\overline{S(\mu ; m)}$. Since $N(S(\mu ; m))$ is the trivial $\mu$-component link, we obtain $Q(S(\mu ; m) ; x)=Q\left(U_{\mu} ; x\right)$ and the following.

Corollary 3.9. $Q\left(L_{(\mu ; m)} ; x\right)=Q\left(U_{\mu} ; x\right)$. 


\section{Trivial Conway polynomial.}

In this section, we show that $K_{m}$ and $L_{(\mu ; m)}$ have trivial Conway polynomial.

The Conway polynomial $\nabla(L ; z) \in \mathbb{Z}[z]$ of an oriented link $L$ is an invariant of the isotopy type of $L$, which is defined by the following formulas:

(C1) $\nabla\left(U_{1} ; z\right)=1$,

(C2) $\nabla\left(L_{+} ; z\right)-\nabla\left(L_{-} ; z\right)=z \nabla\left(L_{0} ; z\right)$,

where $L_{+}, L_{-}$and $L_{0}$ are three links that are identical except near one point where they are as in Figure 13. We call $\left(L_{+}, L_{-}, L_{0}\right)$ a skein triple.

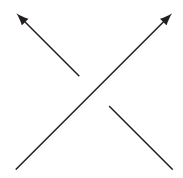

$L_{+}$

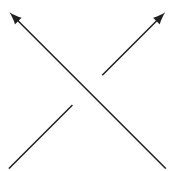

$L_{-}$
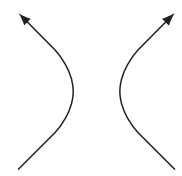

$L_{0}$

Figure 13. A skein triple.

There is a well known formula on the Conway polynomial.

Proposition 4.1 ([3], [11]). Let $T$ and $S$ be properly oriented 2-string tangles. Then,

$$
\nabla(N(T+S) ; z)=\nabla(N(T) ; z) \nabla(D(S) ; z)+\nabla(D(T) ; z) \nabla(N(S) ; z) .
$$

Using Proposition 4.1, we have the following.

Corollary 4.2. Let $m$ and $\mu$ be integers with $m \geq 1$ and $\mu \geq 2$.

(1) $\nabla\left(K_{m} ; z\right)=1$,

(2) $\nabla\left(L_{(\mu ; m)} ; z\right)=0$.

Proof. First, we prove that $\nabla\left(K_{m} ; z\right)=1$. Since $K_{m}$ is defined by $N(R(m)+W)$ and $N(R(m))$ is trivial, Proposition 4.1 gives

$$
\nabla\left(K_{m} ; z\right)=\nabla(D(W) ; z)+\nabla(D(R(m)) ; z) \nabla(N(W) ; z) .
$$

The claim is verified by the facts $\nabla(D(W) ; z)=1$ and $\nabla(N(W) ; z)=0$ derived from Remark 2.1.

Next, we show that $\nabla\left(L_{(\mu ; m)} ; z\right)=0$. Since $L_{(\mu ; m)}$ is defined by $N(S(\mu ; m)+W)$ and $N(S(\mu ; m))$ is the trivial $\mu$-component link, Proposition 4.1 gives

$$
\nabla\left(L_{(\mu ; m)} ; z\right)=\nabla(D(S(\mu ; m)) ; z) \nabla(N(W) ; z) .
$$

Since $\nabla(N(W) ; z)=0$, we have the claim. 
Here, we mention a fact on the Jones polynomial of the link $L_{(\mu ; m)}$.

REMARK 4.3. The Jones polynomial of the link $L_{(\mu ; m)}$ does not depend on the orientations of components of the link because the linking number of any 2-component sublink of the link is zero.

\section{Classification of links.}

In this section, we classify the knots $K_{m}, m \geq 1$, and the links $L_{(\mu ; m)}, \mu \geq 2, m \geq 1$, by using the HOMFLY polynomial.

The HOMFLY polynomial $P(L ; v, z) \in \mathbb{Z}\left[v^{ \pm 1}, z^{ \pm 1}\right]$ of an oriented link $L$ is an invariant of the isotopy type of $L$, which is defined by the following formulas:

(P1) $P\left(U_{1} ; v, z\right)=1$,

(P2) For a skein triple $\left(L_{+}, L_{-}, L_{0}\right)$,

$$
v^{-1} P\left(L_{+} ; v, z\right)-v P\left(L_{-} ; v, z\right)=z P\left(L_{0} ; v, z\right) .
$$

ExAMPLE 5.1. The HOMFLY polynomial of the knot $K_{2}$ introduced in Example 2.2 is given below.

$$
\begin{aligned}
P( & \left.K_{2} ; v, z\right) \\
= & \left(-2 v^{4}+7 v^{2}-2-12 v^{-2}+12 v^{-4}+3 v^{-6}-7 v^{-8}+2 v^{-10}\right) \\
& +z^{2}\left(-5 v^{4}+18 v^{2}-9-32 v^{-2}+32 v^{-4}+9 v^{-6}-18 v^{-8}+5 v^{-10}\right) \\
& +z^{4}\left(-4 v^{4}+17 v^{2}-10-31 v^{-2}+31 v^{-4}+10 v^{-6}-17 v^{-8}+4 v^{-10}\right) \\
& +z^{6}\left(-v^{4}+7 v^{2}-5-13 v^{-2}+13 v^{-4}+5 v^{-6}-7 v^{-8}+v^{-10}\right) \\
& +z^{8}\left(v^{2}-1-2 v^{-2}+2 v^{-4}+v^{-6}-v^{-8}\right) .
\end{aligned}
$$

Let $G$ be a $2 \times 2$ matrix whose entries are in $\mathbb{Z}\left[v^{ \pm 1}, z^{ \pm 1}\right]$ defined by

$$
\left(\begin{array}{cc}
-P\left(U_{2} ; v, z\right) & 1 \\
1 & -P\left(U_{2} ; v, z\right)
\end{array}\right) .
$$

Lickorish and Millett show the following formula on the HOMFLY polynomial for the numerator of addition of two tangles.

Proposition 5.2 ([19]). Let $T$ and $S$ be properly oriented 2-string tangles. Then,

$$
\begin{aligned}
& \left(1-P\left(U_{2} ; v, z\right)^{2}\right) P(N(T+S) ; v, z) \\
& \quad=(P(N(S) ; v, z) P(D(S) ; v, z)) G\left(\begin{array}{l}
P(N(T) ; v, z) \\
P(D(T) ; v, z)
\end{array}\right) .
\end{aligned}
$$

We have the following on the HOMFLY polynomial for the denominator of vertical addition of two tangles. 
Proposition 5.3. Let $T$ and $S$ be properly oriented 2-string tangles. Then, the links $D(T * S)$ and $N(T+S)$ are mutant each other, which, in particular, implies

$$
P(D(T * S) ; v, z)=P(N(T+S) ; v, z) .
$$

Proof. Let $\rho(S)$ be the tangle obtained from $S$ by the mutation which rotates $S$ through angle $\pi / 180^{\circ}$ around an axis perpendicular to the projection plane. Then, the links $D(T * S)$ and $D(T * \rho(S))$ are mutant each other. Since the link $D(T * \rho(S))$ is equivalent to the link $N(T+S)$, we have the result.

REMARK 5.4. Since the link $N(T * S)$ is a connected sum $N(T) \sharp N(S)$ of links $N(T)$ and $N(S)$, it is clear that $P(N(T * S) ; v, z)=P(N(T) ; v, z) P(N(S) ; v, z)$.

We denote by $T(2, n)$ the torus link of type $(2, n)$ whose diagram is represented by $D\left(E_{1 /-n}\right)$. Suppose that $T(2, n)$ is oriented such that two strings of the tangle $E_{1 /-n}$ have parallel orientations. Then, $T(2, n)$ has $|n|$ positive (resp. negative) crossings if $n>0$ (resp. $n<0)$. We also suppose that the tangle $H(m, n), m, n \in \mathbb{N}$, is oriented as introduced in Section 2.

LEMma 5.5. For positive integers $m$ and $n$,

(1) $P(N(H(m, n)) ; v, z)=P(T(2, m-n) ; v, z)$,

(2) $P(D(H(m, n)) ; v, z)=P(T(2, m) ; v, z) P(T(2,-n) ; v, z)$.

Proof. By the definition of $H(m, n)$, we have $N(H(m, n))=N\left(E_{1 /-m}+E_{1 / n}\right)$. The link $N\left(E_{1 /-m}+E_{1 / n}\right)$ is equivalent to $D\left(E_{1 /(m-n)}\right)$ which denotes $T(2, m-n)$. Hence, we obtain the first equality. It is easy to see that $D(H(m, n))$ is equivalent to $D\left(E_{1 /-m}\right) \sharp D\left(E_{1 / n}\right)$ which denotes $T(2, m) \sharp T(2,-n)$. Thus, we have the second equality.

The HOMFLY polynomial for $D(R(m))$ can be represented as follows.

Lemma 5.6. For a positive integer $m$,

$$
\begin{aligned}
\left(1-P\left(U_{2} ; v, z\right)^{2}\right) & P(D(R(m)) ; v, z) \\
= & P(T(2, m) ; v, z) P(T(2,-(m+1)) ; v, z) \\
& +P(T(2,-m) ; v, z) P(T(2, m+1) ; v, z) \\
& -P\left(U_{2} ; v, z\right)\left(1+\prod_{k=m}^{m+1} P(T(2, k) ; v, z) P(T(2,-k) ; v, z)\right) .
\end{aligned}
$$

Proof. Recall that $R(m)=H(m, m+1) * H(m+1, m)$. Since it is shown by Lemma 5.5 that $P(N(H(m, m+1)) ; v, z)=P(N(H(m+1, m)) ; v, z)=1$, Propositions 5.2 and 5.3 give

$$
\left(1-P\left(U_{2} ; v, z\right)^{2}\right) P(D(R(m)) ; v, z)
$$




$$
\begin{aligned}
& ={ }^{t}\left(\begin{array}{l}
P(N(H(m+1, m)) ; v, z) \\
P(D(H(m+1, m)) ; v, z)
\end{array}\right) G\left(\begin{array}{l}
P(N(H(m, m+1)) ; v, z) \\
P(D(H(m, m+1)) ; v, z)
\end{array}\right) \\
& ={ }^{t}\left(\begin{array}{c}
1 \\
P(D(H(m+1, m)) ; v, z)
\end{array}\right) G\left(\begin{array}{c}
1 \\
P(D(H(m, m+1)) ; v, z)
\end{array}\right) .
\end{aligned}
$$

Applying Lemma 5.5 to the last expression of the equality above, we have the lemma.

REMARK 5.7. Since $N(R(m))$ is trivial, it is clear that $P(N(R(m)) ; v, z)=1$.

Here, we introduce a terminology for a Laurent polynomial in two variables $v$ and $z$. A two variable polynomial $g(v, z) \in \mathbb{Z}\left[v^{ \pm 1}, z^{ \pm 1}\right]$ can be written as

$$
g(v, z)=\sum_{j \in \mathbb{Z}} g_{j}(v) z^{j}
$$

where $g_{j}(v) \in \mathbb{Z}\left[v^{ \pm 1}\right]$ and $g_{j}(v)=0$ except a finite number of $j$. The maximal degree of $g(v, z)$ in $z$ denoted by $\max \operatorname{deg}_{z} g(v, z)$ is defined by

$$
\max _{\operatorname{deg}_{z}} g(v, z)= \begin{cases}0 & \text { if } g(v, z)=0 \\ \max \left\{j ; g_{j}(v) \neq 0\right\} & \text { otherwise }\end{cases}
$$

EXAMPLE 5.8. The HOMFLY polynomial for the knot $K_{2}$ is given in Example 5.1.

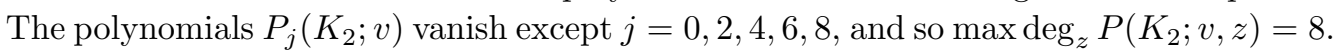

Lemma 5.9. For an integer $n, \max \operatorname{deg}_{z} P(T(2, n) ; v, z)=|n|-1$.

Proof. Since $P(T(2,0) ; v, z)=\left(v^{-1}-v\right) z^{-1}$, the claim is true for the case $n=0$. If $n<0$, then we have $P(T(2, n) ; v, z)=P\left(T(2,|n|) ;-v^{-1}, z\right)$ because $T(2, n)$ is the mirror image of $T(2,|n|)$. It follows that

$$
\begin{aligned}
\max \operatorname{deg}_{z} P(T(2, n) ; v, z) & =\max \operatorname{deg}_{z} P\left(T(2,|n|) ;-v^{-1}, z\right) \\
& =\max \operatorname{deg}_{z} P(T(2,|n|) ; v, z) .
\end{aligned}
$$

Hence, it is sufficient to show the case where $n$ is positive. We prove by induction on the number $n$. If $n \leq 4$, then the claim is verified by direct calculation. Suppose that $n>4$. Combining skein relations for three skein triples $(T(2, n), T(2, n-2), T(2, n-1))$, $(T(2, n-1), T(2, n-3), T(2, n-2))$ and $(T(2, n-2), T(2, n-4), T(2, n-3))$, we have

$$
P(T(2, n) ; v, z)=v^{2}\left(z^{2}+2\right) P(T(2, n-2) ; v, z)-v^{4} P(T(2, n-4) ; v, z) .
$$

Since $\max \operatorname{deg}_{z} P(T(2, n-2) ; v, z)=n-3$ and $\max \operatorname{deg}_{z} P(T(2, n-4) ; v, z)=n-5$ by inductive hypothesis, it is easy to see that $\max \operatorname{deg}_{z} P(T(2, n) ; v, z)=n-1$. This completes the proof.

REMARK 5.10. $P(T(2, n) ; v, z)$ does not vanish because $P\left(L ; v, v^{-1}-v\right)=1[\mathbf{1 9}]$ for a link $L$.

We easily obtain the following by Lemmas 5.5 and 5.9 and Remark 5.10. 
Lemma 5.11. For positive integers $m$ and $n, \max \operatorname{deg}_{z} P(D(H(m, n)) ; v, z)=m+$ $n-2$.

LeMma 5.12. Let $m$ be an integer with $m>1$. Then,

$$
\max \operatorname{deg}_{z} P(D(R(m)) ; v, z)=4 m-3 .
$$

Proof. By Lemma 5.9 and Remark 5.10, we have

$$
\begin{aligned}
\max _{z} \operatorname{deg}_{z} & P(T(2, m) ; v, z) P(T(2,-(m+1)) ; v, z)) \\
& =\max \operatorname{deg}_{z} P(T(2, m) ; v, z)+\max _{z} \operatorname{deg}_{z} P(T(2,-(m+1)) ; v, z) \\
& =2 m-1, \\
\max _{\operatorname{leg}} & (P(T(2, m+1) ; v, z) P(T(2,-m) ; v, z)) \\
& =\max \operatorname{deg}_{z} P(T(2, m+1) ; v, z)+\max \operatorname{deg}_{z} P(T(2,-m) ; v, z) \\
& =2 m-1,
\end{aligned}
$$

and

$$
\begin{aligned}
\max \operatorname{deg}_{z} & \left(\prod_{k=m}^{m+1} P(T(2, k) ; v, z) P(T(2,-k) ; v, z)\right) \\
& =4 m-2
\end{aligned}
$$

Since $4 m-3>2 m-1$ for $m>1$, by Lemma 5.6, we obtain

$$
\begin{aligned}
\max \operatorname{deg}_{z} P(D(R(m)) ; v, z) & =\max _{2} \operatorname{deg}_{z}\left(\left(1-P\left(U_{2} ; v, z\right)^{2}\right) P(D(R(m)) ; v, z)\right) \\
& =4 m-3,
\end{aligned}
$$

completing the proof.

Lemma 5.13. Let $W$ be the tangle depicted in Figure 9. Then,

$$
\max \operatorname{deg}_{z} P(D(W) ; v, z)=4 .
$$

Proof. By Remark 2.1, $D(W)$ is the knot $16_{n 491778}$. Since

$$
\begin{aligned}
P(D(W) ; v, z)=( & \left.v^{2}-2 v^{-2}+2 v^{-4}+v^{-6}-v^{-8}\right) \\
& +z^{2}\left(v^{2}-2-3 v^{-2}+3 v^{-4}+2^{-6}-v^{-8}\right) \\
& +z^{4}\left(-1-v^{-2}+v^{-4}+v^{-6}\right),
\end{aligned}
$$

the lemma is true.

Now we are ready to classify the knots $K_{m}, m \geq 1$.

Proposition 5.14. For a positive integer $m$, $\max \operatorname{deg}_{z} P\left(K_{m} ; v, z\right)=4 m$. 
Proof. If $m=1$, then we see that $K_{m}=N(R(m)+W)$ is equivalent to $D(W)$. The claim is verified by Lemma 5.13. Suppose that $m>1$. By Proposition 5.2 and Remarks 2.1 and 5.7, we have

$$
\begin{aligned}
& \left(1-P\left(U_{2} ; v, z\right)^{2}\right) P\left(K_{m} ; v, z\right) \\
& =P(D(W) ; v, z)+P(D(R(m)) ; v, z) P\left(U_{2} ; v, z\right) \\
& \quad-P\left(U_{2} ; v, z\right)\left(P\left(U_{2} ; v, z\right)+P(D(R(m)) ; v, z) P(D(W) ; v, z)\right) .
\end{aligned}
$$

By Lemmas 5.12 and 5.13 and the equality $\max \operatorname{deg}_{z} P\left(U_{2} ; v, z\right)=-1$, we see that

$$
\begin{array}{rl}
\max \operatorname{deg}_{z} & P\left(K_{m} ; v, z\right) \\
& =\max \operatorname{deg}_{z}\left(\left(1-P\left(U_{2} ; v, z\right)^{2}\right) P\left(K_{m} ; v, z\right)\right) \\
& =\max \operatorname{deg}_{z}\left(P\left(U_{2} ; v, z\right) P(D(R(m)) ; v, z) P(D(W) ; v, z)\right) \\
& =-1+(4 m-3)+4=4 m .
\end{array}
$$

ExAmple 5.15. Proposition 5.14 claims $\max \operatorname{deg}_{z} P\left(K_{2} ; v, z\right)=8$. It is confirmed by Example 5.8 .

The following is an immediate consequence of Proposition 5.14.

Corollary 5.16. Let $m$ and $n$ be positive integers. If $m \neq n$, then the knot $K_{m}$ is distinct from the knot $K_{n}$.

Next, we classify the links $L_{(\mu ; m)}, \mu>1, m \geq 1$. Since $S(2 ; m)=H(2 m, 2 m)$, the following is easily obtained from Lemma 5.11.

Lemma 5.17. For a positive integer $m, \max \operatorname{deg}_{z} P(D(S(2 ; m)) ; v, z)=4 m-2$.

Proposition 5.18. For a positive integer $m, \max \operatorname{deg}_{z} P\left(L_{(2 ; m)} ; v, z\right)=4 m+1$.

Proof. Lemma 5.5 implies $P(N(S(2 ; m)) ; v, z)=P\left(U_{2} ; v, z\right)$. Since $N(W)$ is the trivial 2-component link, we obtain $P(N(W) ; v, z)=P\left(U_{2} ; v, z\right)$. These equalities and Proposition 5.2 provide

$$
\begin{aligned}
& \left(1-P\left(U_{2} ; v, z\right)^{2}\right) P\left(L_{(2 ; m)} ; v, z\right) \\
& \quad \begin{array}{l}
=P\left(U_{2} ; v, z\right)(P(D(W) ; v, z)+P(D(S(2 ; m)) ; v, z)) \\
\quad-P\left(U_{2} ; v, z\right)\left(P\left(U_{2} ; v, z\right)^{2}+P(D(S(2 ; m)) ; v, z) P(D(W) ; v, z)\right) .
\end{array}
\end{aligned}
$$

By Lemmas 5.13 and 5.17 and the equality $\max \operatorname{deg}_{z} P\left(U_{2} ; v, z\right)=-1$, we obtain

$$
\begin{array}{rl}
\max \operatorname{deg}_{z} & P\left(L_{(2 ; m)} ; v, z\right) \\
& =\max \operatorname{deg}_{z}\left(\left(1-P\left(U_{2} ; v, z\right)^{2}\right) P\left(L_{(2 ; m)} ; v, z\right)\right) \\
& =\max \operatorname{deg}_{z}\left(P\left(U_{2} ; v, z\right) P(D(S(2 ; m)) ; v, z) P(D(W) ; v, z)\right) \\
& =-1+(4 m-2)+4=4 m+1 .
\end{array}
$$


Corollary 5.19. Let $m$ and $n$ be positive integers. If $m \neq n$, then the two links $L_{(2 ; m)}$ and $L_{(2 ; n)}$ are distinct.

The link $L_{(\mu ; m)}, \mu \geq 3$, has three types of 2-component sublinks which are $L_{(2 ; m)}$, $L_{(2 ; 1)}$ and the trivial 2-component link. Since Proposition 5.18 shows that $L_{(2 ; m)}$ and $L_{(2 ; 1)}$ are distinct if $m>1$, the following comes from Corollary 5.19.

Corollary 5.20. Let $m, n$ and $\mu$ be integers with $m, n \geq 1$ and $\mu \geq 3$. If $m \neq n$, then the two links $L_{(\mu ; m)}$ and $L_{(\mu ; n)}$ are distinct.

\section{Primeness.}

In this section, we discuss the primeness of the knot $K_{m}$ and the link $L_{(\mu ; m)}$.

A tangle $T=(B, t)$, which is not the trivial 1-string tangle, is prime if it has the following properties:

(1) any 2 -sphere in $B$ which intersects $t$ in two point transversely bounds a 3 -ball in $B$ which intersects $t$ in a trivial arc,

(2) any proper disk in $B$ does not split $t$ in $B$,

(3) any proper disk in $B$ which intersects $t$ transversely in a single point divides $T$ into two tangles, at least one of which is the trivial 1-string tangle.

These properties are called locally trivial, non-split and indivisible in numerical order.

Nakanishi $[\mathbf{2 2}]$ shows the following on the primeness of a 2-string tangle.

Proposition 6.1. Any locally trivial non-split tangle, the number of whose arcs is at most two, is a prime tangle.

By Proposition 6.1, to prove that a 2-string tangle is prime, we only have to check that the tangle has such two properties.

LEMma 6.2. For a positive integer $m$, the tangle $H(2 m, 2 m)$ is prime.

Proof. The tangle $H(2 m, 2 m)$ consists of two arcs and a circle component $K$ which is the trivial knot. Suppose that $H(2 m, 2 m)$ is split. Then, there exists a properly embedded disk in the tangle so that $K$ and at least one of two arcs can be separated by the disk. It follows that the link $D(H(2 m, 2 m))$ should have a split sublink which includes $K$. However, sublinks of $D(H(2 m, 2 m))$ including $K$ are $T(2,2 m)$ and $T(2,-2 m)$ only. Each of them is non-split. This is a contradiction. Hence, $H(2 m, 2 m)$ is non-split. Next, we show that $H(2 m, 2 m)$ is locally trivial. There exists no 2 -sphere in the tangle which bounds a 3-ball containing a knotted arc by the triviality of the arcs and $K$. If there is a 2 -sphere in the tangle which bounds a 3 -ball containing $K$, then we have an arc outside the ball because the sphere intersects the tangle in two points. It follows that the link $D(H(2 m, 2 m))$ should have a split sublink which includes $K$. It leads to a contradiction as stated above. 
Lemma 6.3. For an integer $m$ with $m>1$, the tangles $H(m, m+1)$ and $H(m+1, m)$ are prime.

Proof. We only prove the primeness of the tangle $H(m, m+1)$ for the case where $m$ is even because we can give the proofs for the remaining cases in a similar manner. $H(m, m+1)$ consists of two arcs. One is a trivial arc and the other is a knotted arc to which the torus knot of type $(2,-(m+1))$ is connected. Suppose that $H(m, m+1)$ is split. Then, there exists a properly embedded disk in the tangle so that the trivial arc and the knotted arc can be separated by the disk. Since the knotted arc is contained in one of the two 3-balls obtained by dividing the tangle by the disk, it should persist as a factor of any link created by gluing a tangle to $H(m, m+1)$. However, the link $N(H(m, m+1))$ is the trivial knot, which does not have a non-trivial torus knot as a factor. This is a contradiction. Hence, $H(m, m+1)$ is non-split. Next, we show that $H(m, m+1)$ is locally trivial. If there exists a 2 -sphere in the tangle which bounds a 3 -ball containing a knotted arc, then the knotted arc should be of the torus knot of type $(2,-(m+1))$ and persist as a factor of any link created by gluing a tangle to $H(m, m+1)$. It leads to a contradiction as stated above. Hence, $H(m, m+1)$ is locally trivial.

Lemma 6.4. The tangle $W$ presented in Figure 9 is prime.

Proof. The tangle $W$ consists of two trivial arcs. It implies that there exists no 2-sphere in the tangle which bounds a 3-ball containing a knotted arc. Hence, $W$ is locally trivial. Next, we show that $W$ is non-split. If the $\operatorname{arcs}$ are split, then $W$ should be a trivial 2-string tangle. Since any sum of two trivial 2-string tangles is a 2-bridge knot or link, $D(W)$ must be a 2-bridge knot, in particular, an alternating knot. But, $D(W)$ is the knot $16_{n 491778}$, which is non-alternating. This is a contradiction.

TheOREM $6.5([\mathbf{1 6}],[\mathbf{2 2}])$. Let $(C, v)$ be a tangle and $D$ be a disk properly embedded in $C$ such that $D$ divides $(C, v)$ into two tangles $(A, s)$ and $(B, t)$. We assume the following.

(1) The numbers of points in $(\partial A-D) \cap v,(\partial B-D) \cap v$ and $D \cap v$ are all greater than or equal to 2 ,

(2) $(A, s)$ is prime,

(3) $(B, t)$ is prime.

Then, $(C, v)$ is prime.

Theorem 6.5 implies that the vertical addition of two prime 2-string tangles is a prime tangle.

Corollary 6.6. Let $m$ and $\mu$ be integers with $m \geq 1$ and $\mu \geq 2$.

(1) The tangle $R(m)$ is prime if $m>1$.

(2) The tangle $S(\mu ; m)$ is prime. 
Proof. We begin with the proof of the case (1). Recall that $R(m)$ is defined by $H(m, m+1) * H(m+1, m)$. Since $H(m, m+1)$ and $H(m+1, m)$ are prime by Lemma 6.3, Theorem 6.5 ensures the primeness of $R(m)$. Next, we prove the case $(2)$. The proof is by induction on the number $\mu$. Since $S(2 ; m)$ is the tangle $H(2 m, 2 m)$ by the definition of $S(\mu ; m)$, Lemma 6.2 shows that $S(2 ; m)$ is prime. Suppose that $\mu>2$. $S(\mu ; m)$ can be regarded as $S(\mu-1 ; m) * H(2,2)$. Since $S(\mu-1 ; m)$ and $H(2,2)$ are prime by inductive hypothesis and Lemma 6.2 respectively, it follows from Theorem 6.5 that $S(\mu ; m)$ is prime. This completes the proof.

A link $L$ is locally trivial if any 2 -sphere in $S^{3}$ which intersects $L$ transversally in two points bounds a 3 -ball intersecting $L$ in a trivial arc. A link is prime if it is locally trivial, non-split and non-trivial.

The following is an important result on the primeness of a link obtained by gluing two tangles.

THEOREM $6.7([\mathbf{1 6}],[\mathbf{2 2}])$. A link obtained from two prime tangles by any tangle sum is prime.

Since the tangle $R(1)$ is a trivial 2-string tangle, $K_{1}=N(R(1)+W)$ represents the knot $D(W)=16_{n 491778}$, which is prime. By Lemma 6.4, Corollary 6.6 and Theorem 6.7, we easily obtain the following.

Corollary 6.8. Let $m$ and $\mu$ be integers with $m \geq 1$ and $\mu \geq 2$. Then, the links $K_{m}$ and $L_{(\mu ; m)}$ are prime.

\section{References}

[1] J. W. Alexander, Topological invariants of knots and links, Trans. Amer. Math. Soc., 30 (1928), 275-306.

[2] R. D. Brandt, W. B. R. Lickorish and K. C. Millett, A polynomial invariant for unoriented knots and links, Invent. Math., 84 (1986), 563-573.

[3] J. H. Conway, An enumeration of knots and links, In: Computational Problems in Abstract Algebra, (ed. J. Leech), Pergamon Press, New York, 1969, 329-358.

[4] S. Eliahou, L. H. Kauffman and M. Thistlethwaite, Infinite families of links with trivial Jones polynomial, Topology, 42 (2003), 155-169.

[ 5 ] P. Freyd, D. Yetter, J. Hoste, W. B. R. Lickorish, K. C. Millett and A. Ocneanu, A new polynomial invariant of knots and links, Bull. Amer. Math. Soc., 12 (1985), 239-246.

[6] C. F. Ho, A new polynomial invariant for knots and links, Abstracts Amer. Math. Soc., 6 (1985), 300, Abstract 821-57-16.

[ 7 ] V. F. R. Jones, Hecke algebra representations of braid groups and link polynomials, Ann. of Math., 126 (1987), 335-388.

[8] T. Kanenobu, Infinitely many knots with the same polynomial invariant, Proc. Amer. Math. Soc., 97 (1986), 158-162.

[9] T. Kanenobu, Examples on polynomial invariants of knots and links, Math. Ann., 275 (1986), $555-572$.

[10] T. Kanenobu, Kauffman polynomials for 2-bridge knots and links, Yokohama Math. J., 38 (1991), 145-154.

[11] L. H. Kauffman, The Conway polynomial, Topology, 20 (1981), 101-108.

[12] L. H. Kauffman, An invariant of regular isotopy, Trans. Amer. Math. Soc., 318 (1990), 417-471. 
[13] M. Kidwell, On the degree of the Brandt-Lickorish-Millett-Ho polynomial of a link, Proc. Amer. Math. Soc., 100 (1987), 755-762.

[14] S. Kinoshita and H. Terasaka, On union of knots, Osaka Math. J., 9 (1957), 131-153.

[15] K. Kodama, KNOT, software for computing invariants for knots and links.

[16] W. B. R. Lickorish, Prime knots and tangles, Trans. Amer. Math. Soc., 267 (1981), 321-332.

[17] W. B. R. Lickorish, A relationship between link polynomials, Math. Proc. Camb. Phil. Soc., 100 (1986), 109-112.

[18] W. B. R. Lickorish, Linear skein theory and link polynomials, Topology Appl., 27 (1987), 265-274.

[19] W. B. R. Lickorish and K. C. Millett, A polynomial invariant of oriented links, Topology, 26 (1987), 107-141.

[20] T. Miyauchi, On the highest degree of absolute polynomials of alternating links, Proc. Japan Acad. Ser. A Math. Sci., 63 (1987), 174-177.

[21] S. Morisson, Program to compute the Kauffman polynomial in the Mathematica package "KnotTheory".

[22] Y. Nakanishi, Primeness of Links, Math. Sem. Notes. Kobe Univ., 9 (1981), 415-440.

[23] J. H. Przytycki and P. Traczyk, Invariants of links of Conway type, Kobe J. Math., 4 (1987), 115-139.

[24] D. Rolfsen, Knots and Links, Mathematics Lecture Series, 7, Publish or Perish Inc., Berkeley, Calif., 1976.

[25] H. Schubert, Die eindeutige Zerlegbarkeit eines Knoten in Primknoten, Sitzungsber. Akad. Wiss. Heidelberg, math.-nat. KI., 3 (1949), 57-104.

[26] M. Thistlethwaite, Links with trivial Jones polynomial, J. Knot Theory Ramifications, 10 (2001), 641-643.

\author{
Yasuyuki MiYazAWA \\ Department of Mathematical Sciences \\ Yamaguchi University \\ Yamaguchi 753-8512, Japan \\ E-mail: miyazawa@yamaguchi-u.ac.jp
}

